\title{
Sharing blueprints for better research
}

\author{
Like software before it, open-source hardware promises to encourage collaborative tinkering \\ and promote access to innovative technologies.
}

When the right research tool is missing from a catalog, scientists need to roll up their sleeves and start building. Developing custom-built devices in the laboratory can be slow. Solutions often remain inaccessible because they are difficult for other labs to reproduce or because commercial versions take too long to become available. Researchers may not want to invest the additional effort needed to fully document their inventions or ensure the robustness of their tools for wide release, but adapting the successful open-source software approach to hardware can speed development and encourage the broadest uptake by the research community, and it is a worthwhile scientific goal.

Software is open source when the source code is provided under a copyright license protecting freedom of use, modification and distribution. Similarly, opensource hardware licenses allow users the ability to create, use and sell objects in addition to modifying and sharing designs. Open-source hardware cannot be patented.

The guiding principle of open-source hardware is to provide clear and well-documented design and instructions. Documentation should include photographs, detailed designs, software and complete parts lists. In many cases, wikis and weblogs allow edits, comments and updates to be organized and archived.

Hardware designers should strive to incorporate components that are commonly available or easy to fabricate. A goal of open-source design is to streamline devices so that they are easy for others to reproduce and modify.

Transparency and reproducibility are core scientific values and are critical for disseminating hardware and encouraging adoption by others. But they are often lost in the push to publish the first results using a new device or in the desire to maintain a competitive edge. Developers are often simply lax about including all the technical details of tool design and construction. Releasing hardware under an open-source license codifies the principle of openness and helps ensure that barriers to access are lowered.

Helping the research community at large is important, but what do individual developers have to gain by releasing complete and accessible hardware designs? As open-source software engineers have done, hardware developers can leverage community expertise for problem solving, which can lead to better and more efficient design. Open-access developers form a small but active and supportive community, and designers are more likely to promote an effort with clear community benefits and involvement than contribute to a closed project.

An open approach encourages collaborative design and avoids reinventing the wheel in multiple laboratories. It can also make it easier to get rapid feedback about early prototypes. Documentation standards are evolving for opensource hardware, and it is reasonable to expect that many other attractive features of open-source software, including modularity, early releases and a high level of integration, will be increasingly adopted in the hardware domain.

Open-source design for hardware has lagged behind that for software partly because of implementation barriers: it is easy to share code but harder to fabricate new objects. These hurdles are disappearing with the rise of cheaper, faster and more flexible prototyping tools. Affordable open-source 3D printers, design tools and integrated circuit platforms let scientists move more efficiently from digital design to physical device and build software-based controls for their hardware.

Still, the movement is only a few years old, and the number of open-source hardware initiatives is limited. Some applied technologies are moving in the opensource hardware direction, but such efforts have yet to enter basic research in a significant way. Some researchers are being inspired by other open-source hardware efforts, however. In this spirit, two Correspondences in this issue (p598 and p599) describe affordable opensource light-sheet microscopes capable of recording three-dimensional movies of living organisms.

Manufacturing mature devices requires a great deal of testing and development to ensure that a product will work reliably, which cannot be expected from most academic efforts. The inability to patent open-source designs will sometimes discourage businesses from commercializing research tools. However, alleviating the dependency on commercial suppliers is important when simple and cheap solutions are not available for researchers with poor resources, for specialized fields with limited budgets, or at the leading edge of hardware design where commercialization may lag behind riskier innovation. The viability of several business models that include open-source hardware is also currently being explored.

Different approaches to hardware development can coexist happily. Choosing an open-source approach has the potential to make development more efficient, elicit greater involvement by the research community and help spread technological innovation. 